\title{
Pathology in Practice
}

In collaboration with the American College of Veterinary Pathologists
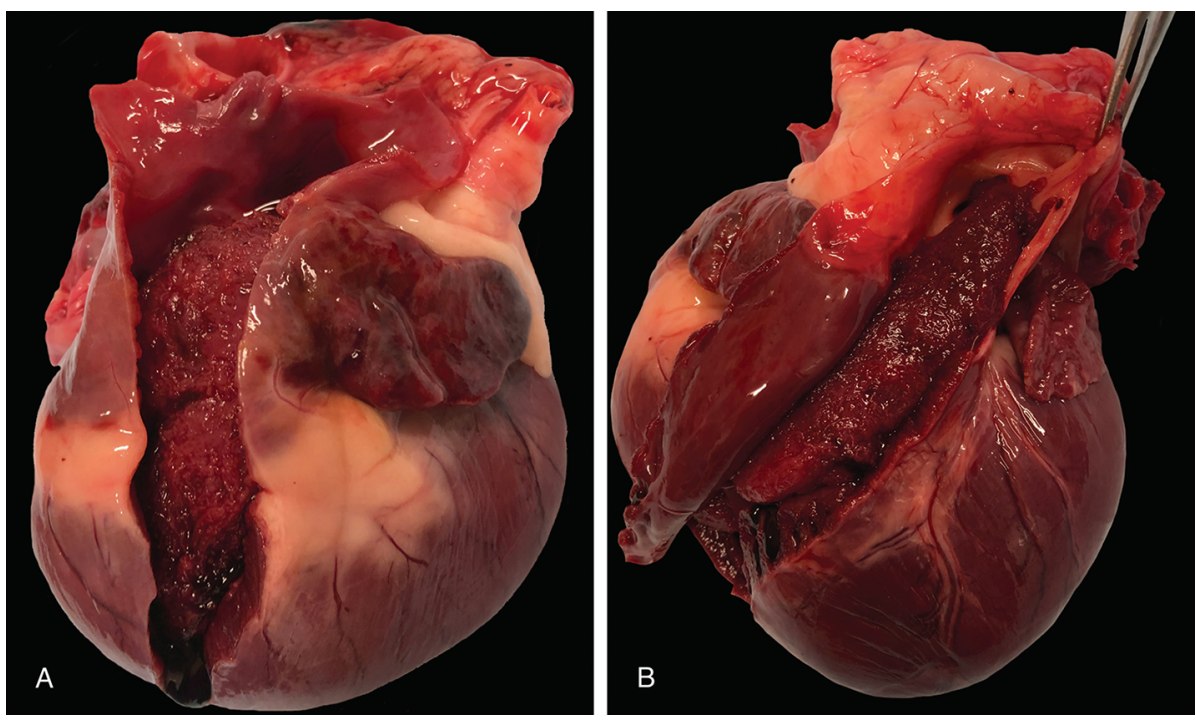

Figure I-Postmortem photographs of the cranial aspect of the cardiac structures opened to view the right atrium and ventricle (A) and right ventricular outflow tract and main pulmonary artery (B) of a 10-year-old spayed female Labrador Retriever mixed-breed dog with a I-week history of hyporexia and lethargy. A-A friable, red mottled mass extends through the right atrium and ventricle. The right atrial appendage is dilated and discolored with red-black mottling. B-The mass extends from the right ventricle to the right ventricular outflow tract, past the pulmonic valve, and into the main pulmonary artery.

\section{History}

A 10 -year-old 17.8 -kg spayed female Labrador Retriever mixed-breed dog was presented to a private practice emergency and specialty center for evaluation because of a 1-week history of hyporexia and lethargy. The dog was completely inappetent for 2 days and vomited once the evening prior to presentation. The dog had eaten part of a plastic bag with chocolate chip cookies 3 days prior, was not receiving any medications, and was up-to-date on vaccination.

\section{Clinical and Gross Findings}

Physical examination revealed a body condition score of $5 / 9,5 \%$ dehydration, and a rectal temperature of $37.4^{\circ} \mathrm{C}$. Mucous membranes were pale, and a capillary refill time could not be detected. Thoracic

Sarah K. Lorbach, DVM'; Tracie D. Romsland, DVM, MS'; Vincent W. Carroll, VMD²; Chloe L. Block, DVM1,3*

\footnotetext{
'Friendship Hospital for Animals, Washington, DC

2Idexx Laboratories, Inc, Westbrook, ME

${ }^{3}$ VCA Bay Area Veterinary Specialists \& Emergency Hospital, San Leandro, CA

*Corresponding author: Dr. Block (chloelblock@gmail.com)

https://doi.org//0.2460/javma.19.09.0433
}

auscultation revealed normal lung sounds, a mild tachycardia ( 140 beats/min), and a grade $2 / 6$ sternal murmur with a diastolic heart sound auscultated on the right. The abdomen was soft and nonpainful on palpation and lacked a detectable fluid wave. Femoral pulses were synchronous but thready. A bedside thoracic and abdominal ultrasonographic evaluation revealed a mass that appeared to be associated with the right heart and mild to moderate hypoechoic abdominal effusion. No abdominal masses were noted. Blood pressure was undetectable with a Doppler ultrasonic flow detector but was $125 / 105 \mathrm{~mm} \mathrm{Hg}$ using an oscillometric device. A CBC revealed (patient value; reference range) a normal Hct (55.6\%; 37.3\% to $61.7 \%)$ with a leukocytosis $(19.95 \mathrm{~K} / \mu \mathrm{L} ; 5.05$ to $16.76 \mathrm{~K} / \mu \mathrm{L})$ comprised of a neutrophilia $(16.58 \mathrm{~K} / \mu \mathrm{L}$; 2.95 to $11.64 \mathrm{~K} / \mu \mathrm{L})$ and monocytosis $(1.61 \mathrm{~K} / \mu \mathrm{L} ; 0.16$ to 1.12$)$. Mild thrombocytopenia $(132 \mathrm{~K} / \mu \mathrm{L} ; 148$ to $484 \mathrm{~K} / \mu \mathrm{L}$ ) was present with platelet aggregates on the peripheral blood smear. Serum biochemical analyses revealed high BUN ( $35 \mathrm{mg} / \mathrm{dL} ; 7$ to $27 \mathrm{mg} / \mathrm{dL}$ ), hyperphosphatemia $(9.2 \mathrm{mg} / \mathrm{dL} ; 2.5$ to $6.8 \mathrm{mg} / \mathrm{dL})$, and high activities of alanine aminotransferase $(248 \mathrm{U} / \mathrm{L} ; 10$ to $125 \mathrm{U} / \mathrm{L})$ and alkaline phosphatase (257 U/L; 23 to $212 \mathrm{U} / \mathrm{L})$. Concentrations of total protein $(6.0 \mathrm{~g} / \mathrm{dL}$; 5.2 to $8.2 \mathrm{~g} / \mathrm{dL})$ and albumin $(3.4 \mathrm{~g} / \mathrm{dL} ; 2.2$ to $3.9 \mathrm{~g} / \mathrm{dL})$ were normal. 
Echocardiography revealed a massive inhomogeneous and irregular mass visible within the right auricle, right atrium, and cranial and caudal vena cavae. It could be seen prolapsing across the tricuspid valve into the right ventricle, wrapping through the right ventricular outflow tract and prolapsing across the pulmonic valve. The right and left pulmonary arteries and left heart appeared small, consistent with volume underloading. No pleural or pericardial effusion was present.

Due to poor prognosis, the owner elected euthanasia. Abdominocentesis and abdominal ultrasonography were planned but aborted based on the echocardiographic results and subsequent decision for euthanasia. Based on the owner's wishes, the postmortem evaluation was limited to cardiac structures. On necropsy, there was a very small amount of clear pericardial effusion considered within normal limits. There was an approximately $9.5-\mathrm{cm}$-long, $1.5-\mathrm{cm}-\mathrm{high}$, and $4.5-\mathrm{cm}$-wide extremely friable red-black mottled mass originating within the right auricle (Figure I). The mass extended into the cranial and caudal vena cavae, through the right atrium and tricuspid valve, into the right ventricle and out the right ventricular outflow tract, and through the pulmonic valve into the main pulmonary artery and right pulmonary artery.

Formulate differential diagnoses, then continue reading.

\section{Cytologic Findings}

An impression smear of the mass revealed a markedly atypical cell population that ranged from oval to spindloid in shape (Figure 2). Moderate to marked anisocytosis and anisokaryosis were present. Cells had round to irregular nuclei with a coarsely stippled chromatin pattern and contained $\geq 1$ to multiple nucleoli of variable size and shape. Low numbers of binucleated and multinucleated cells were found, often with variably sized nuclei within the same cell. The cytoplasm was moderate in amount and medium blue. Mitotic figures were easily found and many of them were bizarre. Low numbers of macrophages were noted. No infectious agents were apparent.

\section{Histopathologic Findings}

Multiple sections of the mass were submitted for histopathology, some including cardiac muscle.

Sections of the right atrium were characterized by an irregularly shaped, poorly delineated, densely cellular neoplastic mass composed of spindle-shaped to stellate cells arranged in tightly packed bundles and sheets, supported by a sparse fibrovascular stroma (Figure 3). The neoplastic cells were sometimes forming ill-defined vascular channels, clefts, and spaces. Individual cells were plump with indistinct cell borders, small to moderate amounts of eosinophilic cytoplasm, and round to oval nuclei containing

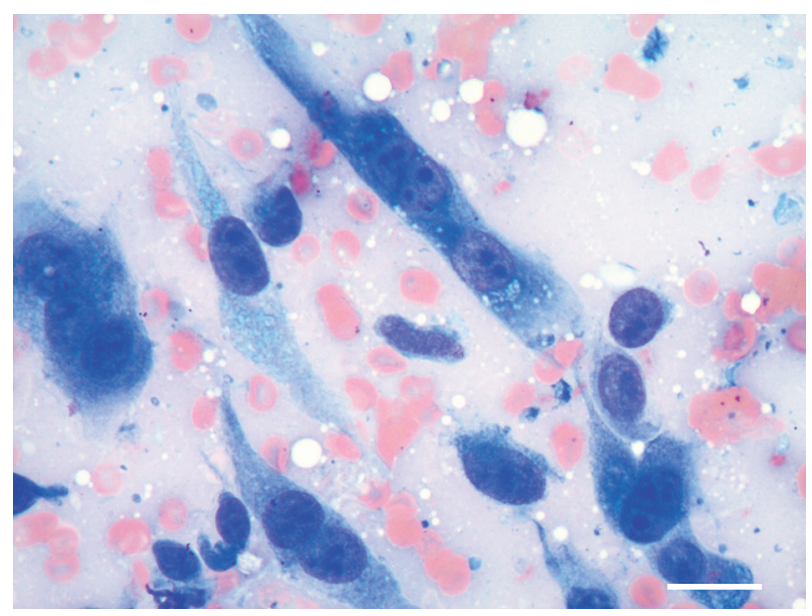

Figure 2-Photomicrograph of an impression smear of the right auricular mass from the dog in Figure I. There are oval to spindloid cells with anisocytosis, anisokaryosis, and irregular nuclei, with bi- and multinucleated cells. Multiple nucleoli are present and have variable sizes and shapes. The cytoplasm is moderate in amounts and medium blue. Wright Giemsa stain; bar $=20 \mu \mathrm{m}$.

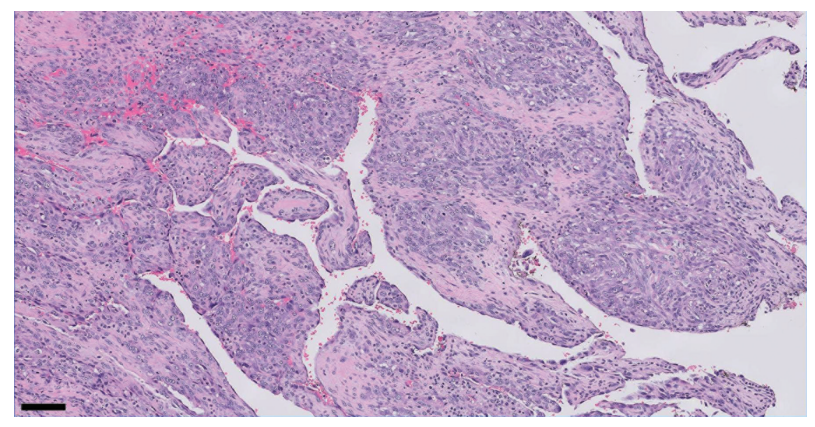

Figure 3-Photomicrograph of a section from the right auricular mass from the dog described in Figure I. Tightly packed bundles and sheets of neoplastic spindle to stellate-shaped cells with sparse supporting fibrovascular stroma are present. A small foci of necrosis is present within the mass in addition to mild neutrophilic and lymphoplasmacytic infiltrates and hemorrhage. H\&E stain; bar $=100 \mu \mathrm{m}$.

coarsely stippled chromatin with 2 to 3 small nucleoli. Anisocytosis and anisokaryosis were moderate to marked and 23 mitotic figures were observed in ten 400X fields (total surface area, $2.37 \mathrm{~mm}^{2}$ ). Occasional binucleate/multinucleate and karyomegalic neoplastic cells were observed. Small foci of necrosis were present within the mass in addition to mild neutrophilic and lymphoplasmacytic infiltrates and hemorrhage.

\section{Morphologic Diagnosis and Case Summary}

Right auricular hemangiosarcoma with extension into and partial occlusion of the cranial and caudal vena cava, right atrium, right ventricle, and main pulmonary artery. 


\section{Comments}

The clinical and antemortem diagnostic findings in this case were consistent with a massive rightsided cardiac mass thought to be hemangiosarcoma (HSA), an extensive thrombus, or another neoplasm. Dogs with cardiac masses can present with a multitude of clinical signs ranging in severity from an incidental finding to nonspecific signs (eg, respiratory symptoms, lethargy, and inappetence) to abdominal distention and sudden collapse. ${ }^{1,2}$ This patient was presented with nonspecific signs including a 1-week history of progressive inappetence, lethargy, and having vomited once.

On auscultation, a grade II/VI systolic sternal murmur was detected in addition to a right-sided extra heart sound, retrospectively consistent with a tumor plop due to the presence of a large intraventricular mass colliding with the ventricular walls throughout the cardiac cycle. Cardiac output was deemed compromised based on the presence of pale mucous membranes, a blood pressure undetectable by a Doppler ultrasonic flow detector, tachycardia, and poor pulse quality. Thoracic ultrasonography was performed to evaluate for obvious causes of decreased cardiac output including pericardial effusion and decreased systolic function. Although neither of these were present, the scan revealed a mass prompting cardiology consultation.

Echocardiography confirmed a massive rightsided intracardiac mass. A tumor was considered most likely, but a large thrombus could not be excluded because there was no clear point of attachment within the heart and the size was substantial. No pericardial effusion was present. A thrombus was thought to be less likely due to the absence of concurrent conditions associated with hypercoagulability or blood stasis.

In dogs, primary HSA is the most common right atrial mass and is 10 times as likely than other cardiac tumors such as those of neuroendocrine origin, thyroid carcinoma, lymphosarcoma, mesothelioma, and fibrosarcoma. ${ }^{3,4}$ Thrombus formation has been described in the vena cavae and the atria of dogs in association with a multitude of predisposing causes and factors including immune-mediated hemolytic disease, sepsis, protein-losing nephropathies, central venous catheter placement, endocrinopathies, neoplasia, and cardiac disease..$^{5-7}$ A recent case report documented a left ventricular thrombus in a dog with nephrotic syndrome related to renal amyloidosis secondary to a thymoma. ${ }^{8}$

Despite adequate intrinsic cardiac function, volume underloading of the left heart and pulmonary vasculature was consistent with signs of obstructive right-sided congestive heart failure (CHF) and dehydration. The abdominal effusion was thought to be a consequence of right-sided CHF given the echocardiographic findings and the hypoechoic nature of the fluid. Other causes (such as hemorrhage secondary to splenic or hepatic hemangiosarcoma) could not entirely be excluded; however, hemorrhage typically causes echogenic fluid, and a brief abdominal scan did not reveal an obvious splenic mass. Cardiology consultation was performed prior to diagnostic abdominocentesis, and based on those results, euthanasia was elected. Unfortunately, a necropsy limited to the cardiac structures only was approved. A full necropsy could have contributed valuable information and strengthened the suspicion of right heart failure.

Cytologically, the tumor was consistent with a sarcoma and histopathology confirmed hemangiosarcoma. Although HSA is the most common neoplasm within the right atrium and auricle, the case described is remarkable due to the intracardiac extensiveness of the tumor in combination with right $\mathrm{CHF}$, and absence of pericardial effusion. Of dogs with cardiac HSA (cHSA), between 83 and 100\% have been found to have pericardial effusion. ${ }^{1,9,10}$ In a study of 9 dogs with cHSA undergoing necropsy, invasion into and rupture of right atrium was noted in 2 dogs. ${ }^{9}$ Given the aggressive nature of this type of tumor, the extension beyond the right atrium and tricuspid valve into the right outflow tract is remarkable.

Canine HSA can be found in any tissue with vascularization, but it is most commonly found to affect the spleen, skin and subcutaneous tissues, liver, and heart. It has also been reported to affect the pericardium resulting in hemorrhagic pericardial effusion. ${ }^{11}$ This aggressive, malignant neoplasm can also be found in cats, horses, cows and sheep. ${ }^{12}$ Although HSA has a propensity to affect the heart, a study of 104 dogs with HSA demonstrated that 65 (63\%) affected the spleen, whereas only $3(0.03 \%)$ affected the heart. ${ }^{13}$ Treatment typically includes a combination of surgery with or without a doxorubicin-based chemotherapeutic protocol. In a study evaluating 23 dogs with surgical resection of cHSA within the right atrium and auricle survival with or without chemotherapy yielded a median survival of 43 to 118 days (range, 0 to 229 days) with the longest being from dogs with right auricular (as compared to right atrial) resection. Survival in those receiving adjuvant chemotherapy ranged from 12 to 228 days. ${ }^{1}$ When dogs with presumptive cHSA were treated with singleagent doxorubicin alone, median survival time was 116 days as compared to 12 days for untreated control dogs. ${ }^{14}$ Surgical removal of right atrial and auricular tumors has been described; however, the tumor in the case of the present report was considered nonsurgical due to its expansion throughout the entire right heart. ${ }^{1,10}$,

\section{Acknowledgments}

Funding for laboratory diagnostics was provided by an educational fund sponsored by Friendship Hospital for Animals. Funding sources did not have any involvement in data analysis and interpretation or writing and publication of the manuscript. The authors declare that there were no conflicts of interest. 


\section{References}

1. Weisse C, Soares N, Beal MW, et al. Survival times in dogs with right atrial hemangiosarcoma treated by means of surgical resection with or without adjuvant chemotherapy: 23 cases (1986-2000). J Am Vet Med Assoc. 2005;226(4):575-579. doi:10.2460/javma.2005.226.575.

2. Rajagopalan V, Jesty SA, Craig LE, et al. Comparison of presump tive echocardiographic and definitive diagnoses of cardiac tumors in dogs. J Vet Intern Med. 2013;27(5):1092-1096. doi:10.1111/jvim.12134.

3. Ware WA, Hopper DL. Cardiac tumors in dogs: 1982-1995. $J$ Vet Intern Med. 1999;13(2):95-103. doi:10.1892/0891-6640 (1999)013\%3C0095:ctid\%3E2.3.co;2.

4. Speltz MC, Maniel JC, Tobias AH, et al. Primary cardiac fibrosarcoma with pulmonary metastasis in a Labrador Retriever Vet Pathol. 2007;44(3):403-407. doi:10.1354/vp.44-3-403.

5. Palmer KG, King LG, VanWinkle TJ. Clinical manifestations and associated disease syndromes in dogs with cranial vena cava thrombosis: 17 cases (1989-1996). J Am Vet Med Assoc. 1998;213(2):220-224

6. Langston CE, Eatroff AE. Hemodialysis catheter-associated fibrin sheath in a dog. J Vet Emerg Crit Care (San Antonio). 2018;28(4):366-371. doi:10.1111/vec.12721.

7. Preston AR, Sullivan LA. Dislodgement of a right atrial thrombus and subsequent pulmonary thromboembolism following tracheal stent deployment in a dog. J Vet Emerg Crit Care (San Antonio). 2016;26(6):809-814. doi:10.1111/vec.12459.

8. Loewen JM, Cianciolo RE, Zhang L, et al. Concurrent renal amyloidosis and thymoma resulting in a fatal ventricular thrombus in a dog. J Vet Intern Med. 2018;32(3):1160-1165. doi:10.1111/jvim. 15062 .

9. Noszczyk-Nowak A, Nowak M, Paslawksa U, et al. A retrospective study of cardiac hemangiosarcoma in dogs. Turk J Vet Anim Sci. 2014;38:77-81. doi:10.3906/vet-1301-42.

10. Ployart S, Libermann S, Doran I, et al. Thoracoscopic resection of right auricular masses in dogs: 9 cases (2003-2011). J Am Vet Med Assoc. 2013;242(2):237-241. doi:10.2460/ javma.242.2.237.

11. DeSandre-Robinson DM, Quina MT, Lurie DM. Pericardial hemangiosarcoma in a 10-year-old papillon. J Am Anim Hosp Assoc. 2018;54(5):e545-04. doi:10.5326/JAAHA-MS-6612.

12. Robinson WF, Robinson NA. Cardiovascular system. In: Maxie G, ed. Jubb, Kennedy \& Palmer's Pathology of Domestic Animals. Elsevier; 2016; 1-101.e1. doi:10.1016/ B978-0-7020-5319-1.00012-8.

13. Brown NO, Patnaik AK, MacEwen G. Canine hemangiosarcoma: retrospective analysis of 104 cases. J Am Vet Med Assoc. 1985;186:56-58.

14. Mullin C, Arkans M, Sammarco C, et al. Doxorubicin chemotherapy for presumptive cardiac hemangiosarcoma in dogs. Vet Comp Oncol. 2016;14(4):e171-e183. doi:10.1111/ vco.12131. 